\title{
Author Correction: One size does not fit all for mutational signatures
}

Giovanni Ciriello (1)

Correction to: Nature Cancer https://doi.org/10.1038/s43018-020-0033-7, published online 17 February 2020.

In the version of this article initially published, the institution for the second affiliation ('Swiss Cancer Center Lausanne') was incorrect. The second affiliation (in full) should be 'Swiss Cancer Center Léman, Lausanne, Switzerland'. The error has been corrected in the HTML and PDF versions of the article.

Published online: 18 March 2020

https://doi.org/10.1038/s43018-020-0051-5

(c) Springer Nature America, Inc. 2020 